EPJ manuscript No.

(will be inserted by the editor)

\title{
Carbonyl sulphide under strong laser field: time-dependent density functional theory
}

\author{
G. Bilalbegović \\ Department of Physics, Faculty of Science, University of Zagreb, \\ Bijenička 32, 10000 Zagreb, Croatia
}

To be published in The European Physical Journal D

\begin{abstract}
The first $52 \mathrm{fs}$ of a time evolution of the electron density in OCS after an interaction with an intense sub 10 fs laser pulse are studied using the time-dependent density functional theory. The nuclear motion in this linear trimer is simulated by the classical molecular dynamics method. Laser fields of intensity $10^{13} \mathrm{~W} / \mathrm{cm}^{2}$ and $10^{15} \mathrm{~W} / \mathrm{cm}^{2}$ are used. Details of the laser induced changes of the structure, as well as the ionization rate are sensitive to the applied field intensity and its polarization. It is found that under suitable conditions the OCS molecule bends soon after an interaction with a laser pulse. A deviation from the linear geometry of up to $23.6^{\circ}$ and charged ions of up to +3 are observed. The time evolution of electric dipole moments and the time-dependent electron localization function (ELF) are also studied.
\end{abstract}

PACS. 31.15.ee Time-dependent density functional theory - 33.80.-b Photon interactions with molecules - 36.40.Qv Stability and fragmentation of clusters

\section{Introduction}

An interaction between nanoparticles and strong laser fields is now an active area of research [12/34. Femtosecond pulsed lasers are able to produce high-intensity fields and they are used to initiate and investigate various processes in nanoparticles 1223 . It is customary to call intensities above $10^{13} \mathrm{~W} / \mathrm{cm}^{2}$ as intense laser fields, and time intervals below $10^{-13} \mathrm{~s}$ as ultra-short laser pulses. Experimental and theoretical studies have been shown that the multi-photon ionization mechanism dominates a lasermolecule interaction at intensities $<10^{14} \mathrm{~W} / \mathrm{cm}^{2}$ for laser frequencies in the near infrared part of the spectrum. The tunnel and multi-electron dissociative ionizations prevail for field intensities greater than $10^{14} \mathrm{~W} / \mathrm{cm}^{2}$. Fields below $10^{13} \mathrm{~W} / \mathrm{cm}^{2}$ are not intense enough to induce the 
multiple ionization of neutral molecules. Research efforts are now oriented towards attosecond laser pulses and intensities of $10^{24} \mathrm{~W} / \mathrm{cm}^{2}$ [4. When atoms, molecules, or clusters, accelerated to a high kinetic energy, impinge on thin foils they sometimes undergo through the process of Coulomb explosion. It is also possible to achieve similar conditions if a nanoparticle collides with an accelerated beam of other particles, or interacts with intense laser fields [5]6]. In these processes often occurs the multiple ionization of a targeted nanoparticle. This nanoparticle then explodes as a result of the strong Coulomb repulsion. One or several charge centers may form in an initial nanoparticle when the loss of electrons occurs under laser light. Products of the Coulomb explosion are high-energy electrons and ions, and under suitable conditions even Xrays. A behavior of fragments in the Coulomb explosion sheds light on interactions in an initial nanoparticle. The Coulomb explosion imaging is successfully used to determine an equilibrium structure of nanoparticles.

The carbonyl sulphide is an important material present in the Earth's atmosphere, volcanic gases, ice cores in Antarctica, and the interstellar medium. It catalyzes the formation of biological molecules and it has been suggested that OCS played an important role in the origin of life on our planet [7]. The charged clusters of carbonyl sulphide have been also studied [89]. In its ground state the OCS molecule is linear, but it is bent in the first excited state [1011]. In addition, the OCS molecule consists of three different atoms. Therefore, in comparison with homonuclear trimers, it is more prone to various mod- ifications of the shape in chemical processes and under strong fields. It has been shown that the OCS molecule at high energies close to dissociation exhibits chaotic dynamics [12. The Coulomb explosion of carbonyl sulphide has been investigated using ion-momentum imaging techniques [13. The laser pulses of $55 \mathrm{fs}$, up to $2 \times 10^{15} \mathrm{~W} / \mathrm{cm}^{2}$ of intensity, and wave length of $\lambda=790 \mathrm{~nm}$ have been used. The shape of the exploding OCS molecule has been determined. Measured ion momentum maps have been compared to those obtained using the Monte Carlo simulation. The ion momentum maps have been shown that $\mathrm{O}^{3+}$ and $\mathrm{S}^{3+}$ exist along the laser polarization direction. The central $\mathrm{C}^{3+}$ exhibits a four-lobe structure which indicates that a non-linear geometry of OCS develops in the Coulomb explosion. A peak bond angle of $170^{\circ}$ and bonds stretching have been measured. The O-C and C-S bonds doubled in an interaction with laser pulses. In a recent work the stronger field of $\simeq 10^{16} \mathrm{~W} / \mathrm{cm}^{2}$ has been used to investigate the multiple ionization of OCS [14]. The three dimensional covariance mapping technique has been applied.

The time-dependent density functional theory (TDDFT) [15]16[17] is a powerful technique for a description of excitations in nanoparticles. It is possible to use this method to study particle dynamics under strong laser pulses [16]18|19|20|21|22]. The time-dependent Schrödinger equation is replaced by the time-dependent Kohn-Sham one (atomic units are used)

$$
i \frac{\partial}{\partial t} \phi_{i}(\mathbf{r}, t)=\left[-\frac{1}{2} \nabla^{2}+v_{K S}(\mathbf{r}, t)\right] \phi_{i}(\mathbf{r}, t) .
$$

In this equation $v_{K S}(\mathbf{r}, t)=v_{H}(\mathbf{r}, t)+v_{x c}(\mathbf{r}, t)+v_{e x t}(\mathbf{r}, t)$, where $v_{H}$ is the Hartree potential, $v_{x c}$ is the exchange- 
correlation potential, and $v_{e x t}$ is an external potential. The particle density $n$ is calculated from

$$
n(\mathbf{r}, t)=\sum_{i}^{N} \phi_{i}^{*}(\mathbf{r}, t) \phi_{i}(\mathbf{r}, t),
$$

where $N$ is the number of occupied states. The laser field enters in an external term of the Kohn-Sham potential. In the classical dipole approximation the potential of the laser field is

$$
V(\mathbf{r}, t)=I f(t) \sin (\omega t) \sum_{i=1}^{N} \mathbf{r} \cdot \alpha,
$$

where $I$ denotes the intensity of the field, $\omega$ is the frequency, and the $\alpha$ is the polarization of the light. The function $f(t)$ determines the shape of the laser pulse. The laser potential is a strong term and it is necessary to solve the Kohn-Sham equations in a non-perturbative regime.

The Born-Oppenheimer approximation is often applied in the analysis of the molecules. It assumes that the motion of nuclei and electrons can be separated. Nuclei are much heavier and therefore their motion often can be neglected when electrons move. However, in some physical situations the Born-Oppenheimer approximation breaks, and it is important to treat the coupled dynamics of electrons and nuclei. One example of a such coupled motion occurs for molecules in strong laser fields. In the calculations presented here nuclei are treated as classical point particles. The Newton equations for the nuclei are solved for the force which is calculated using the Ehrenfest's theorem 2324. Electrons are described by the quantum TDDFT. They are exposed to the Coulomb field of classical nuclei and the laser field. This approach produces errors when the molecule dissociates under a strong laser light. In the theoretical analysis of the laser-molecule interaction it is difficult to treat on the same level situations where the particles in the molecule are bonded together and where nuclear densities are delocalized. The classical treatment of nuclei brakes when a probability for a dissociation of the molecule in the laser field is high [17/25]. The proper treatment of the coupling between electronic and nuclear motion under strong laser light should be carried out using the multi-component TDDFT [26 27]. In this theory both nuclei and electrons are treated as quantum particles. However, this approach is still in development. The multi-component TDDFT is computationally time consuming and it has been performed only for small systems.

In this work mixed techniques of the quantum mechanical time-dependent density functional theory and the classical dynamics method are used to study the heteronuclear linear trimer OCS after an irradiation by an intense and short laser pulse. The results show a bending of the molecule and an elongation of its bonds, as well as ionizations, intensive energy exchanges and charge redistributions. In the following the computational method is described in Sec. II. Results and discussion are presented in Sec. III. A summary and conclusions are given in Sec. IV.

\section{Computational method}

The Coulomb explosion of the carbonyl sulphide is studied by TDDFT method. Calculations are performed using the Octopus code [23], where a real-space, pseudopotential based treatment of the TDDFT has been developed. 
Physical quantities are expanded using a mesh in the real space. In addition, the ground state properties of OCS are also studied by the pseudopotential and planewave based Abinit code [28. The Troullier-Martins pseudopotentials [29] are used in both simulations, and the OCS molecule is positioned in the parallelepiped box with the side of 30 a.u. A Cartesian grid with the constant mesh spacing of $0.2 \AA$ is applied in simulations based on the Octopus code.

The ground-state of OCS obtained using the density functional theory is perturbed by the laser pulse. The timedependent Kohn-Sham equations are integrated using the time step of $\Delta t=0.002 \hbar / \mathrm{eV}=1.32 \times 10^{-18} \mathrm{~s}$. The time evolution of up to $52.8 \mathrm{fs}$ is followed. This is a long time for the TDDFT method. The approximated enforced timereversal symmetry algorithm is applied to treat the evolution operator in the time-dependent Kohn-Sham problem [30]. The adiabatic local density approximation exchange functional in the parametrization of Perdew and Wang is chosen [31. In this work the velocity Verlet algorithm is used to follow the classical time evolution of atomic cores. The OCS molecule is oriented along the $\mathrm{z}$ axis and exposed to the linearly polarized laser light along the $\mathrm{x}, \mathrm{y}$, or $\mathrm{z}$ axis.

Both O-C-S and S-C-O orientations towards an incoming laser pulse are studied for the light polarized along the molecule axis. The absorbing conditions are used at the boundaries of the box 32 .

Electronic excitation spectra of carbonyl sulphide have been measured from 5 to $360 \mathrm{eV}$ using dipole spectroscopy [33]34. The first absorption peak is rather broad and its maximum is at $7-8 \mathrm{eV}$. Using TDDFT it is possible to calculate the absorption spectrum as $\sigma(\omega) \sim|d(\omega)|^{2}$, where $d(\omega)$ is the Fourier transform of the induced dipole moment $d(t)=\int z n(\mathbf{r}, t) d^{3} r$, and $n(\mathbf{r}, t)=|\psi(\mathbf{r}, t)|^{2}$ is an electron density. The absorption spectrum of OCS, calculated using the Octopus code, shows that the first peak is at $7 \mathrm{eV}$. This is in a good agreement with experiments 33 34. Simulations of the laser-molecule interaction are done at the nonresonant frequency of $2.5 \mathrm{eV}$, i.e., below the first absorption peak. Experimental studies of the Coulomb explosion in OCS have been performed at the nonresonant frequency of $\lambda=790 \mathrm{~nm}=1.57$ $\mathrm{eV}$ [1314. The length gauge, pulses with a cosine envelope, a total length of $6.6 \mathrm{fs}$, and maximal intensities of $I_{1}=1.3272 \times 10^{13} \mathrm{~W} / \mathrm{cm}^{2}$ and $I_{2}=1.3272 \times 10^{15} \mathrm{~W} / \mathrm{cm}^{2}$ are applied in these simulations.

\section{Results and discussion}

The ground state density functional theory calculation of the OCS molecule produces a good agreement with experimental results. The molecule is linear, the $\mathrm{S}-\mathrm{C}$ distance is $1.548 \AA$, whereas the $\mathrm{O}-\mathrm{C}$ one is $1.148 \AA$. The measured values of these distances are $1.561 \AA$ and $1.156 \AA$, respectively [35]. The results for changes of the geometry when the laser field is polarized perpendicularly to the initial molecule axis are shown in Table I. The $\mathrm{C}-\mathrm{S}$ distance starts to change at $3.96 \mathrm{fs}$, the $\mathrm{O}-\mathrm{C}$ distance at $9.24 \mathrm{fs}$, and the angle $\angle(\mathrm{O}-\mathrm{C}-\mathrm{S})$ at $14.52 \mathrm{fs}$, for the laser intensity $I_{1}$. The $\mathrm{O}-\mathrm{C}$ distance first increases up to $1.209 \AA$ (at $19.14 \mathrm{fs}$ ), and then decreases. For example, the O-C distance is $1.164 \AA$ at 26.40 fs. The $\mathrm{C}-\mathrm{S}$ distance 
increases up to $1.689 \AA$ at 31.68 fs and then decreases. The results for the intensity $I_{2}=1.3272 \times 10^{15} \mathrm{~W} / \mathrm{cm}^{2}$ also show that the distances do not change monotonically in time. The distances between atoms when the laser field is polarized along the initial molecule axis are presented in Table II. There the molecule is linear. In this Table the results for two orientations of the molecule towards a laser pulse are shown. It is calculated that data for these two orientations differ only quantitatively. The results for the $\mathrm{S}-\mathrm{C}-\mathrm{O}$ orientation are presented in examples for an interaction with the z-polarized laser light in Figs. 2-5. Figure 1 shows an example of the change of the $\mathrm{O}-\mathrm{C}$ and C-S atomic distances during the 52.8 fs of the time evolution. Similar nonmonotonic changes of the internuclear distance have been recently measured and calculated in the $\mathrm{D}_{2}^{-}$molecule study by ultrashort laser fields on a time scale of 3 ps [36].

In general, the angle between bonds changes when the laser polarization is along directions which are perpendicular to the molecular axis. OCS preserves its linear geometry when the laser light is polarized along the molecule axis. However, bending of OCS induced by pulses polarized along the internuclear axis may occur on a longer time scale, i.e., after $52.8 \mathrm{fs}$. The observed maximum of the angle between bonds is $23.6^{\circ}$ and it is calculated for the x-polarized pulse of intensity $I_{2}=1.3272 \times 10^{15} \mathrm{~W} / \mathrm{cm}^{2}$. As in experiments [1314, it is found that the $\mathrm{O}-\mathrm{C}$ bond is stronger than the $\mathrm{C}-\mathrm{S}$ bond, and that both bonds often strongly increase after an interaction with a laser pulse. It is already known that atomic bonds are substantially

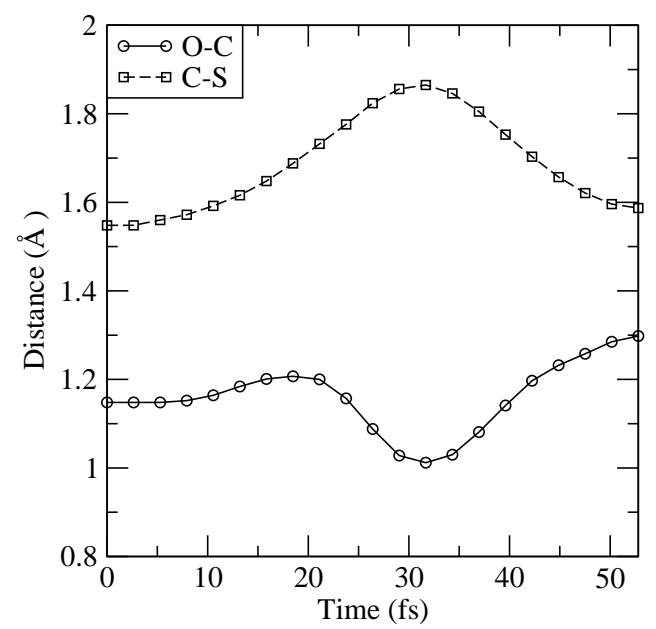

Fig. 1. The time evolution of atomic distances after an interaction with the laser light of $I_{2}=1.3272 \times 10^{15} \mathrm{~W} / \mathrm{cm}^{2}, \mathrm{x}$ polarization.

elongated in comparison with equilibrium ones in the processes of an enhanced multiple ionization and the Coulomb explosion 37.

The OCS molecule ejects electrons after an interaction with a strong enough laser pulse. Figure 2 shows how the electronic charge of this 16-valence electron molecule (as represented by the pseudopotential model) changes when OCS is irradiated by a laser pulse. As shown in Figs. 2(b), charge states of up to +3 are observed under the field of $\sim 10^{15} \mathrm{~W} / \mathrm{cm}^{2}$. It is also found that for all laser light polarizations the electronic charge in the box does not change (within the simulation time of $52.8 \mathrm{fs}$ ) under the laser field of $I_{1}=1.3272 \times 10^{13} \mathrm{~W} / \mathrm{cm}^{2}$. The results show that the z-polarized laser light is more efficient in removal of electrons from the OCS molecule. In this simulation only the overall electronic charge of the molecule is calculated by integrating the electron density in the simulation box. However, in the experimental work and cor- 
Table 1. The time evolution of the structure for laser intensities $I_{1}=1.3272 \times 10^{13} \mathrm{~W} / \mathrm{cm}^{2}$ and $I_{2}=1.3272 \times 10^{15} \mathrm{~W} / \mathrm{cm}^{2}$, using the polarization direction perpendicular to the initial axis of the molecule (x polarization). The distances d (here, in Table II and Fig. 1) are geometrical separations between atoms (including the cases where they are not chemically bonded).

\begin{tabular}{ccccccc}
\hline \multicolumn{5}{c}{$I_{1}$} & \multicolumn{5}{c}{$I_{2}$} \\
\hline time $[\mathrm{fs}]$ & $\mathrm{d}(\mathrm{O}-\mathrm{C})$ & $\mathrm{d}(\mathrm{C}-\mathrm{S})$ & $\angle(\mathrm{O}-\mathrm{C}-\mathrm{S})$ & $\mathrm{d}(\mathrm{O}-\mathrm{C})$ & $\mathrm{d}(\mathrm{C}-\mathrm{S})$ & $\angle(\mathrm{O}-\mathrm{C}-\mathrm{S})$ \\
\hline 2.64 & 1.148 & 1.548 & $180^{\circ}$ & 1.148 & 1.548 & $180^{\circ}$ \\
7.92 & 1.148 & 1.560 & $180^{\circ}$ & 1.152 & 1.572 & $180^{\circ}$ \\
13.20 & 1.172 & 1.576 & $180^{\circ}$ & 1.184 & 1.616 & $179.2^{\circ}$ \\
18.48 & 1.204 & 1.604 & $178.7^{\circ}$ & 1.207 & 1.688 & $170.8^{\circ}$ \\
26.40 & 1.164 & 1.668 & $166.9^{\circ}$ & 1.088 & 1.824 & $171.7^{\circ}$ \\
31.68 & 1.049 & 1.689 & $171.7^{\circ}$ & 1.012 & 1.865 & $176.9^{\circ}$ \\
\hline
\end{tabular}

Table 2. The time evolution of the structure for the laser intensity $I_{2}=1.3272 \times 10^{15} \mathrm{~W} / \mathrm{cm}^{2}$ and a laser pulse polarized along the internuclear axis (z polarization). The data for two orientations (S-C-O and O-C-S) of the carbonyl sulphide molecule in the laser field are presented.

\begin{tabular}{ccccccc}
\hline \multicolumn{5}{c}{$\mathrm{O}-\mathrm{C}-\mathrm{O}-\mathrm{C}-\mathrm{S}$} \\
\hline time [fs] & $\mathrm{d}(\mathrm{O}-\mathrm{C})$ & $\mathrm{d}(\mathrm{C}-\mathrm{S})$ & $\angle(\mathrm{O}-\mathrm{C}-\mathrm{S})$ & $\mathrm{d}(\mathrm{O}-\mathrm{C})$ & $\mathrm{d}(\mathrm{C}-\mathrm{S})$ & $\angle(\mathrm{O}-\mathrm{C}-\mathrm{S})$ \\
\hline 2.64 & 1.148 & 1.548 & $180^{\circ}$ & 1.148 & 1.552 & $180^{\circ}$ \\
7.92 & 1.184 & 1.548 & $180^{\circ}$ & 1.192 & 1.568 & $180^{\circ}$ \\
13.20 & 1.260 & 1.572 & $180^{\circ}$ & 1.272 & 1.600 & $180^{\circ}$ \\
18.48 & 1.336 & 1.648 & $180^{\circ}$ & 1.288 & 1.660 & $180^{\circ}$ \\
26.40 & 1.288 & 1.844 & $180^{\circ}$ & 1.260 & 1.784 & $180^{\circ}$ \\
31.68 & 1.176 & 2.008 & $180^{\circ}$ & 1.252 & 1.880 & $180^{\circ}$ \\
\hline
\end{tabular}

responding Monte Carlo simulations several $\left(Q_{1}, Q_{2}, Q_{3}\right)$ fragmentation channels have been proposed, where the labels are as in $\left(O^{Q_{1}+}, C^{Q_{2}+}, S^{Q_{3}+}\right)$ [1314]. For example, it has been found that $(3,3,5)$ is a channel with the highest ionization under the field of $\simeq 10^{16} \mathrm{~W} / \mathrm{cm}^{2}$.

Several typical examples of the kinetic and potential energy are presented in Figs. 3 and 4 . It is well known that positively charged ions form in the process of the laser- molecule interaction. The Coulomb repulsion of the ions and an initial binding energy of the molecule are stored as the potential energy. The repulsive Coulomb potential energy is strong and it is rapidly released as a kinetic energy of the molecule fragments. The results show that energies change after a time delay of at least 2 fs. Regularly positioned peaks are clearly visible in Fig. 3(b). The peaks are less pronounced under conditions of Fig. 3(a,c). 


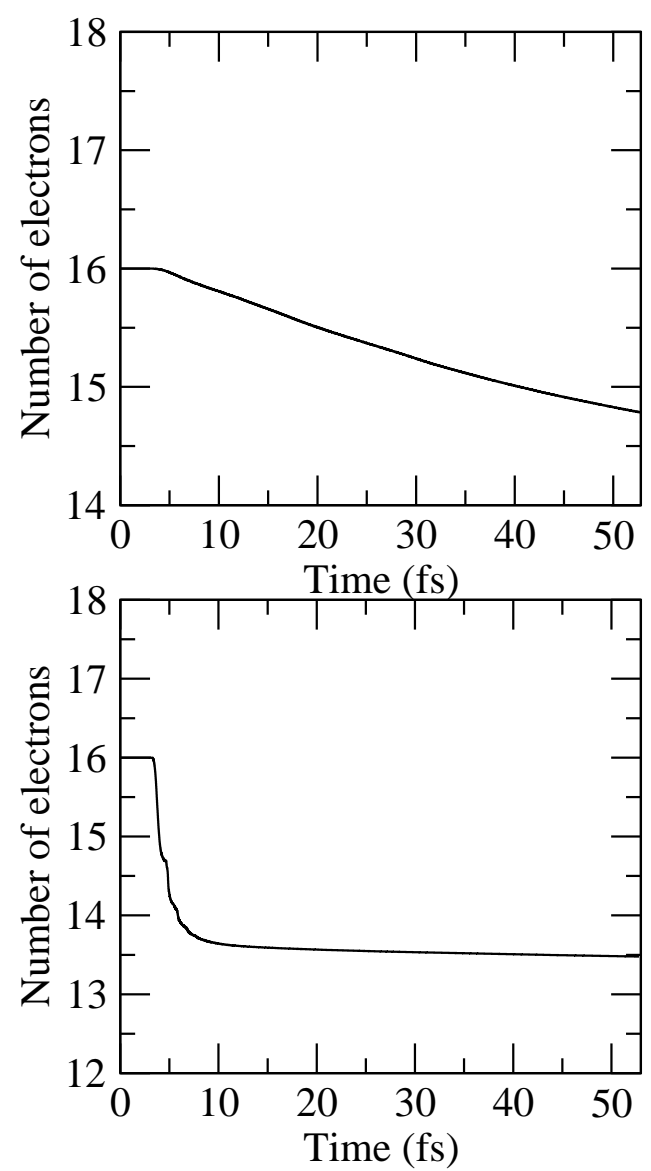

(a)

Fig. 2. The time evolution of the electronic charge after an interaction with the laser light: (a) $I_{2}=1.3272 \times 10^{15} \mathrm{~W} / \mathrm{cm}^{2}$, x polarization, (b) $I_{2}=1.3272 \times 10^{15} \mathrm{~W} / \mathrm{cm}^{2}$, z polarization.

Fig. 3. The time evolution of the kinetic energy of OCS after an interaction with a laser pulse: (a) $I_{1}=1.3272 \times 10^{13} \mathrm{~W} / \mathrm{cm}^{2}$, z polarization, (b) $I_{2}=1.3272 \times 10^{15} \mathrm{~W} / \mathrm{cm}^{2}, \mathrm{x}$ polarization, (c) $I_{2}=1.3272 \times 10^{15} \mathrm{~W} / \mathrm{cm}^{2}$, z polarization.

Therefore, the peak shapes and positions are intensity and polarization dependent. These peaks in the time evolution of the kinetic energy and similar ones for the potential energy (Fig. 4(a,b)) show that an intensive redistribution of the energy occurs in the OCS molecule after an interaction with a laser pulse. Under conditions of Fig. 3(b) the laser field caused the molecule to bend. Then, the molecule starts to vibrate and the result is an oscillatory exchange of kinetic and potential energy. The potential energy (Fig. $4(\mathrm{c}))$ is much larger in an absolute value, and therefore the peaks are more visible in the kinetic energy (Fig. 3(b)). It has been measured for OCS perturbed by the laser light that the kinetic energy release varies with the ionization channels 14]. This study has been performed using laser pulses focused to $\sim 10^{16} \mathrm{~W} / \mathrm{cm}^{2}$ and the kinetic energies between $4.1 \mathrm{eV}$ and $105 \mathrm{eV}$ have been measured. In the ground state of the molecule its potential energy is negative. When the molecule is perturbed by the laser light, the potential energy increases. Figure 4 shows that the potential energy is always negative. Therefore, the system preserves at least the part of its initial binding energy. The time evolution of the electric dipole moment $d_{z}(t)$ of the molecule is calculated and presented in Fig. 5. When the light is polarized along the molecule axis, the change of an electric dipole distribution in the first several fs is especially intensive and approximately follows the oscillatory features of the laser pulse.

The electron localization function (ELF) has become an important tool for a description of chemical bonds in molecules and solids [38]39. The results of many studies have been shown that, in comparison with electron densities, the ELFs better describe the properties of localized and delocalized electrons in atoms and their aggregates. Recently the time-dependent electron localization function suitable for an analysis of the creation and breaking of bonds has been introduced [21. The three-dimensional representations of the time-dependent ELF isosurfaces for 
Fig. 6. The time-dependent electron localization function (ELF). An initial ground-state structure is shown in (a). The same molecule after an interaction with the $\mathrm{x}$-polarized laser pulse of the intensity $I_{2}=1.3272 \times 10^{15} \mathrm{~W} / \mathrm{cm}^{2}$ : (b) $0.66 \mathrm{fs}$, (c) $2.64 \mathrm{fs}$.

OCS are presented in Fig. 6. The comparison of ELFs in the ground-state (Fig. 6(a)) and after 0.66 fs (Fig. 6(b)) shows that the instability of the OCS molecule first occurs at the sulfur atom (the upper left part of the ELF shown in Fig. 6(b)). Figure 6(b) represents the point of the system time evolution when the distances are $d(\mathrm{O}-$ $\mathrm{C})=1.148 \AA, \mathrm{d}(\mathrm{C}-\mathrm{S})=1.548 \AA$. Further analysis of the time-dependent ELF confirms that the electron ejection begins around the $\mathrm{S}$ atom. Figure 6(c) shows several blobs of a negative charge. The corresponding geometry of the molecule shown in Fig. $6(\mathrm{c})$ is $\mathrm{d}(\mathrm{O}-\mathrm{C})=1.148 \AA$, $\mathrm{d}(\mathrm{C}-\mathrm{S})=$ $1.560 \AA$ After 2.64 fs electrons are already ejected from the molecule.

\section{Conclusions}

An electron-ion dynamics of the carbonyl-sulphide molecule exposed to the intense, sub $10 \mathrm{fs,} \mathrm{linearly} \mathrm{polarized} \mathrm{laser}$ pulse is studied using calculations based on the numerical integration of the equations developed within the timedependent density functional theory. The dynamics is followed for 52.8 fs. The results of this simulation show that the OCS molecule bends during the ionization process if the laser polarization direction is perpendicular to the initial axis of the molecule. This molecule preserves its lin- ear morphology after 52.8 fs of the time evolution when it is oriented along the laser polarization direction. An intensive redistribution of charges and energies, and the stretching of bonds are observed. These results are in a good agreement with experimental studies of the carbonyl sulphide molecule in a strong laser field [1314. However, experiments have been carried out on a much longer time scale. TDDFT is an ab initio computational method which is able to simulate processes in materials at nanoscales induced by short and strong laser pulses.

This work has been carried out under the HR-MZOS project "Electronic Properties of Surfaces and Nanostructures". I am grateful to the University Computing Center SRCE for their help and computer time.

\section{References}

1. K. Yamanouchi, Science 295, 1659 (2002)

2. J.P. Marangos, S. Baker, N. Kajumba, J.S. Robinson, J.W.G. Tisch, R. Torres, Phys. Chem. Chem. Phys. 10, $35(2008)$

3. J.P. Marangos, Molecules in a strong laser field, lecture notes, unpublished (2004)

4. P.B. Corkum, F. Krausz, Nature Physics 3, 381 (2007)

5. L.J. Frasinski, K. Codling, P. Hatherly, J. Barr, I.N. Ross, W.T. Toner, Phys. Rev. Lett. 58, 2424 (1987)

6. T.E. Dermota, Q. Zhong, A.W. Castleman, Chem. Rev. 104, $1861(2004)$

7. L. Leman, L. Orgel, M.R. Ghadiri, Science 306, 283 (2004)

8. E. Surber, A. Sanov, J. Chem. Phys. 118, 9192 (2003)

9. G. Bilalbegović, Chem. Phys. Lett. 441, 309 (2007) 
Fig. 4. The time evolution of the potential energy of OCS after an interaction with a laser pulse: (a) $I_{1}=1.3272 \times 10^{13}$ $\mathrm{W} / \mathrm{cm}^{2}$, x polarization, (b) $I_{1}=1.3272 \times 10^{13} \mathrm{~W} / \mathrm{cm}^{2}, \mathrm{z}$ polarization, (c) $I_{2}=1.3272 \times 10^{15} \mathrm{~W} / \mathrm{cm}^{2}, \mathrm{x}$ polarization, $(\mathrm{d})$ $I_{2}=1.3272 \times 10^{15} \mathrm{~W} / \mathrm{cm}^{2}, \mathrm{z}$ polarization.

Fig. 5. The time-dependent dipole moment (along the molecule axis) after an interaction with a laser pulse: $\left(\right.$ a) $I_{1}=1.3272 \times 10^{13}$ $\mathrm{W} / \mathrm{cm}^{2}$, x polarization, (b) $I_{1}=1.3272 \times 10^{13} \mathrm{~W} / \mathrm{cm}^{2}, \mathrm{z}$ polarization, (c) $I_{2}=1.3272 \times 10^{15} \mathrm{~W} / \mathrm{cm}^{2}, \mathrm{x}$ polarization, $(\mathrm{d})$ $I_{2}=1.3272 \times 10^{15} \mathrm{~W} / \mathrm{cm}^{2}, \mathrm{z}$ polarization.

10. T. Suzuki, H. Katayanagi, S. Nanbu, M. Aoyagi, J. Chem. Phys. 109, 5778 (1998)

11. A. Sugita, M. Mashino, M. Kawasaki, Y. Matsumi, R. Bersohn, G. Trott-Kriegeskorte, K.H. Gericke, J. Chem. Phys. 112, 7095 (2000)

12. R. Paskauskas, C. Chandre, T. Uzer, Phys. Rev. Lett. 100, $083001(2008)$

13. J.H. Sanderson, T.R.J. Goodworth, A. El-Zein, W.A. Bryan, W.R. Newell, A.J. Langley, P.F. Taday, Phys. Rev. A 65, $043403(2002)$

14. W.A. Bryan, W.R. Newell, J.H. Sanderson, A.J. Langley, Phys. Rev. A 74, 053409 (2006)

15. E. Runge, E.K.U. Gross, Phys. Rev. Lett. 52, 997 (1984)

16. C. Fiolhais, F. Nogueira, M. Marques, eds., A Primer in Density Functional Theory (Springer, Berlin, 2003)

17. K. Burke, J. Werschnik, E.K.U. Gross, J. Chem. Phys. 123, 062206 (2005)

18. A. Castro, M.A.L. Marques, J.A. Alonso, G.F. Bertsch, A. Rubio, Eur. Phys. J. D 28, 211 (2004)

19. A.G. Urena, K. Gasmi, S. Skowronek, A. Rubio, P.M. Echenique, Eur. Phys. J. D 28, 193 (2004)

20. M. Isla, J.A. Alonso, Phys. Rev. A 72, 023201 (2005)

21. T. Burnus, M.A.L. Marques, E.K.U. Gross, Phys. Rev. A 71, 010501(R) (2005)
22. E. Suraud, P.G. Reinhard, Phys. Rev. Lett. 85, 2296 $(2000)$

23. A. Castro, H. Appel, M. Oliviera, C.A. Rozzi, X. Andrade, F. Lorenzan, M.A.L. Marques, E.K.U. Gross, A. Rubio, Phys. Stat. Sol. (b) 243, 2465 (2006)

24. F. Calvayrac, P.G. Reinhard, E. Suraud, C.A. Ullrich, Phys. Reports 337, 493 (2000)

25. T. Kreibich, R. van Leeuwen, E.K.U. Gross, Chemical Phys. 304, 183 (2004)

26. T. Kreibich, E.K.U. Gross, Phys. Rev. Lett. 86, 2984 $(2001)$

27. O. Butriy, H. Ebadi, P.L. de Boeij, R. van Leeuwen, E.K.U. Gross, Phys. Rev. A 76, 052514 (2007)

28. X. Gonze, J.M. Beuken, R. Caracas, F. Detraux, M. Fuchs, G.M. Rignanese, L. Sindic, M. Verstraete, G. Zerah, F. Jollet et al., Comput. Mat. Sci. 25, 478 (2002)

29. N. Troullier, J.L. Martins, Phys. Rev. B 43, 1993 (1991)

30. A. Castro, M.A.L. Marques, A. Rubio, J. Chem. Phys. 121, $3425(2004)$

31. J.P. Perdew, Y. Wang, Phys. Rev. B 45, 13244 (1992)

32. T. Fevens, H. Jiang, SIAM J. Sci. Comput. 21, 255 (1999)

33. R. Feng, G. Cooper, C.E. Brion, Chem. Phys. 252, 359 $(2000)$

34. Spectral atlas of gaseous molecules, http://www . atmosphere.mpg.de/enid/2295 
35. E. Surber, S. Ananthavel, A. Sanov, J. Chem. Phys. 116, $1920(2002)$

36. B. Feuerstein, T. Ergler, A. Rudenko, K. Zrost, C.D. Schroter, R. Moshammer, J. Ullrich, T. Niederhausen, U. Thumm, Phys. Rev. Lett. 99, 153002 (2007)

37. T. Seideman, M.Y. Ivanov, P.B. Corkum, Phys. Rev. Lett. 75, $2819(1995)$

38. A.D. Becke, N.E. Edgecombe, J. Chem. Phys. 92, 5397 (1990)

39. A. Savin, R. Nesper, S. Wengert, T.F. Fässler, Angew. Chem., Int. Ed. Engl. 36, 1808 (1997) 
This figure "Fig3.jpeg" is available in "jpeg" format from: http://arxiv.org/ps/0806.4369v1 
This figure "Fig4.jpeg" is available in "jpeg" format from: http://arxiv.org/ps/0806.4369v1 
This figure "Fig5.jpeg" is available in "jpeg" format from: http://arxiv.org/ps/0806.4369v1 
This figure "Fig6.jpeg" is available in "jpeg" format from: http://arxiv.org/ps/0806.4369v1 